\title{
"DimSUb" APLICACIÓN PARA EL DISEÑO Y DIMENSIONADO DE SUBUNIDADES DE RIEGO LOCALIZADO
}

\author{
Arviza Valverde, Jaime (1). Balbastre Peralta, Ibán (2), Palau Estevan, Carmen \\ Virginia (3)
}

${ }^{1}$ Universitat Politècnica de València. Dpto. Ingeniería Rural. jarviza@agf.upv.es
${ }^{2}$ Universitat Politècnica de València. Dpto. Ingeniería Rural. ibbalpe@agf.upv.es
${ }^{3}$ Universitat Politècnica de València. Dpto. Ingeniería Rural. virpaes@agf.upv.es

\section{Resumen}

DimSub es una aplicación informática desarrollada con Visual Basic for Application (VBA) en el entorno Excel cuyo objetivo es el diseño y dimensionado de subunidades de riego localizado admitiendo múltiples opciones: Emisores auto compesantes y no compensantes; alimentación del lateral y terciaria tanto por extremo, punto medio y punto intermedio; doble lateral por fila de plantas en terciarias y posibilidad de terciarias telescópicas. Además, admite varios materiales para la terciaria y el usuario puede modificar todas estas variables. También, permite la gestión de ficheros de datos, calcula el estado de mediciones y los resultados pueden exportarse a RGW2015 para su posterior dimensionado de la red de transporte.

Se ha utilizado un único formulario para facilitar el manejo de la aplicación y que todas las opciones de dimensionado y cálculo se seleccionen en la misma, y que los resultados se visualicen de forma conjunta para una correcta interpretación. soluciones.

Para cada caso estudiado cuantifica las mediciones y los costes de las distintas

\section{Abstract}

DimSub is a software application developed with Visual Basic for Application (VBA) in the Excel whose objective is the design and sizing of drip irrigation subunits admitting multiple options: auto regulated drippers and non regulated ones. Feeding lateral and maninfold by extreme, midpoint or an intermediate point. Lateral double row of plants in manifolds and double diameter manifold possibility. It supports various materials for manifold and the user can modify all these variables. Allows management of data files, enables to calculate measurements and results can be exported to RGW2015 for subsequent sizing the pressure distribution network.

A unique userform has been used to run all options in order to improve the use of the application. Each alternative can be studied in terms of costs and measures. 


\section{Introducción y objetivos.}

El uso generalizado de tecnologías de riego a presión no ha llevado asociado el desarrollo de herramientas de diseño y dimensionado acordes a las necesidades de técnicos e ingenieros, y que garanticen que las soluciones propuestas den respuesta a las necesidades planteadas en estas obras de infraestructura.

En el caso en particular de los sistemas de riego localizado, debido en gran medida a las dimensiones discretas de las parcelas, y por otra parte debido a determinadas políticas comerciales de fabricantes y distribuidores a que no se le preste al diseño y dimensionado el rigor que requiere.

En riego localizado la subunidad de riego o conjunto de laterales conectadas a una terciaria y controlados por un elemento de regulación de presión ya sea manual o automático es la parte fundamental del diseño de los sistemas de riego localizado, en tanto en cuanto, el objetivo principal de cualquier sistema de riego es alcanzar la máxima eficiencia de aplicación, y ello pasa por garantizar una alta uniformidad de distribución en subunidad.

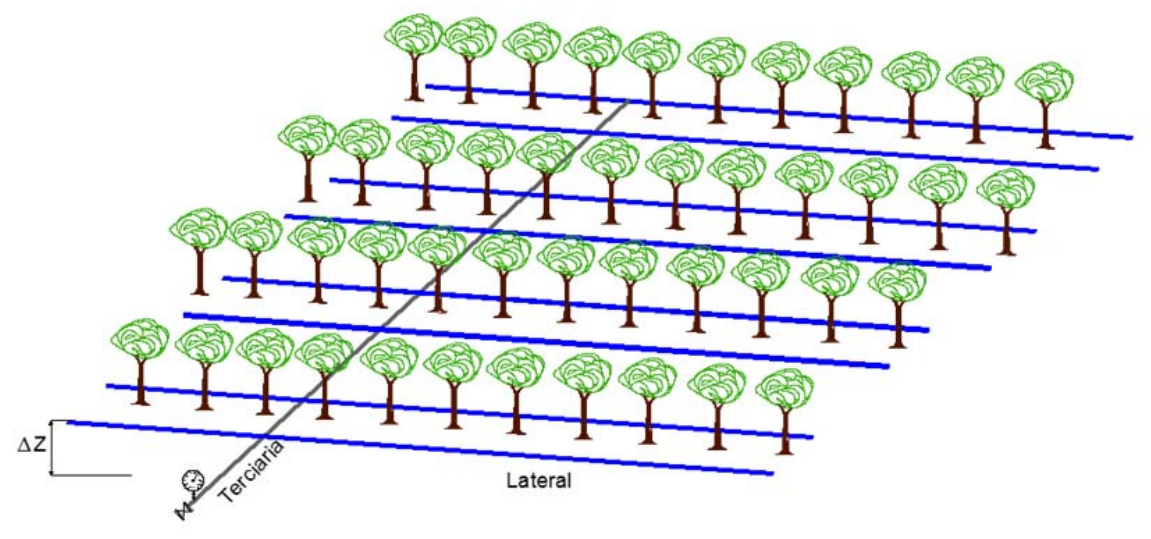

Figura 1: Subunidad de riego

El objetivo que se ha planteado es desarrollar una aplicación informática de distribución libre que permite abordar el diseño y dimensionado de subunidades de riego con el mayor rigor posible, considerando la alta casuística que se presenta en lo que se refiere a tipos de emisores, alimentación de lateral y terciaria, y disposición de emisores en lateral y laterales en la terciaria.

\section{Materiales y métodos}

Dado que la aplicación iba a ser de libre distribución se planteó utilizar el entono de Excel de Microsoft, software comercial universalmente difundido, desarrollando una serie de formularios mediante Visual Basic for Application (VBA) 
La metodología de cálculo de subunidades, ya sea para emisores no compensantes como para emisores auto compensantes se ilustran en las siguientes figuras.

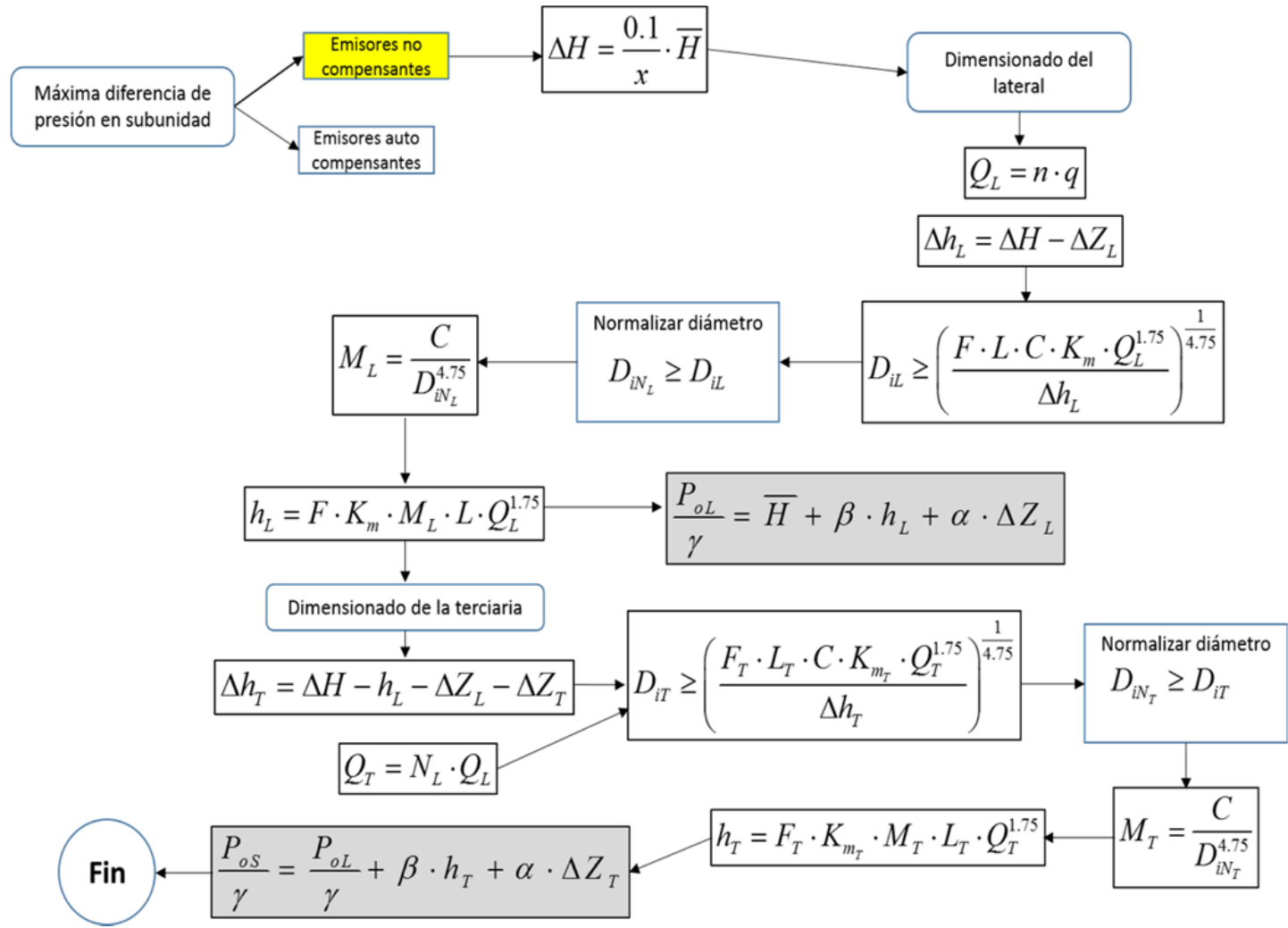

Figura 2: Diagrama para el dimensionado de una subunidad con emisores no compesantes.

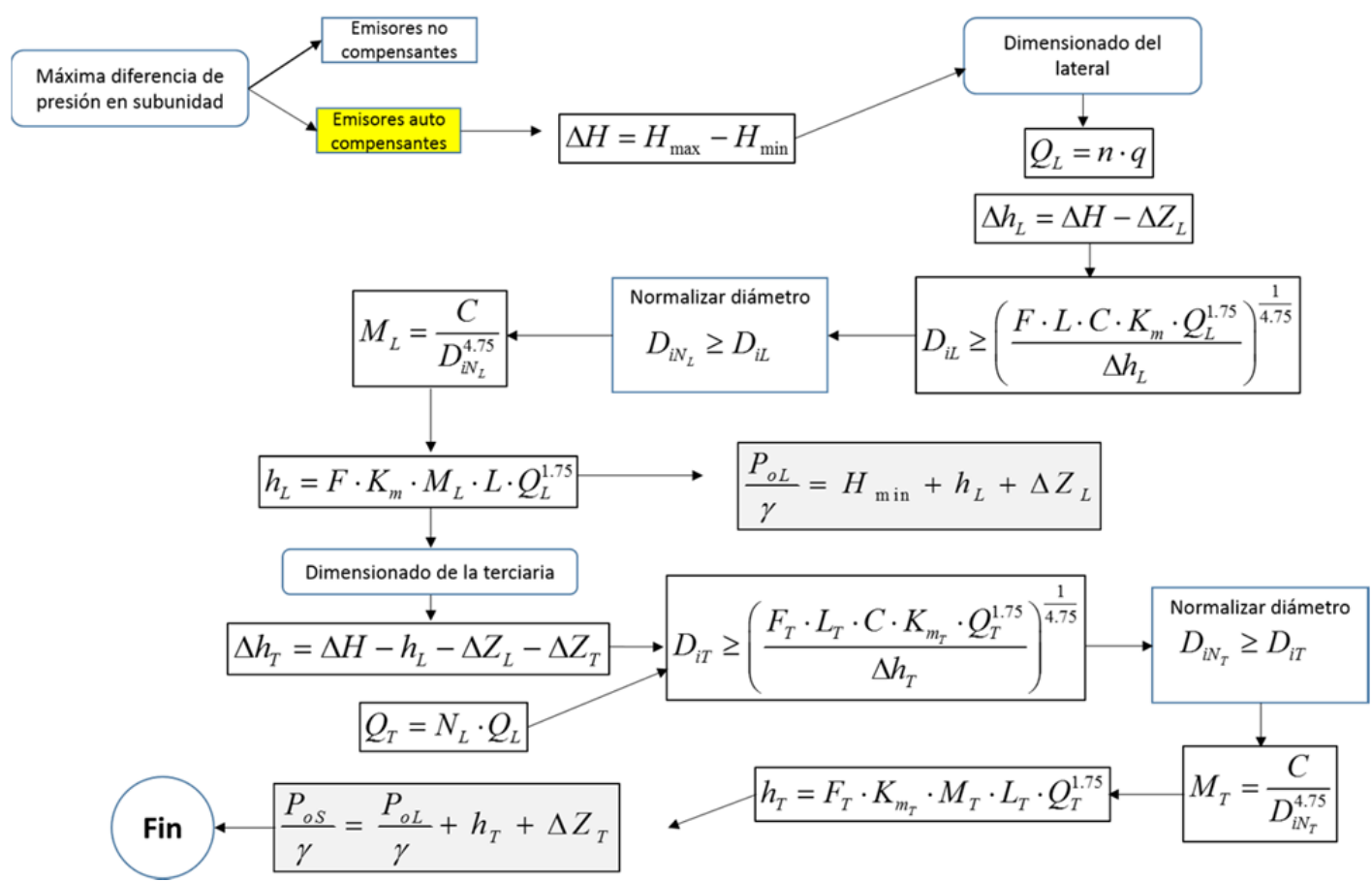

Figura 3: Diagrama de dimensionado para una subunidad con emisores auto compensantes

Cuando tanto lateral o terciaria tiene desnivel descendente, la presión mínima no se verifica al final del lateral, por lo que ha sido necesario desarrollar los correspondientes algoritmos para la determinación del punto de presión mínima en 
lateral y terciaria, y poder proceder a posteriori a el correcto cálculo de la variación máxima de presión en ambas tuberías.

En el caso de laterales y terciarias de dimensiones importantes y con desniveles significativos se ha contemplado la posibilidad de alimentación por un punto intermedio.

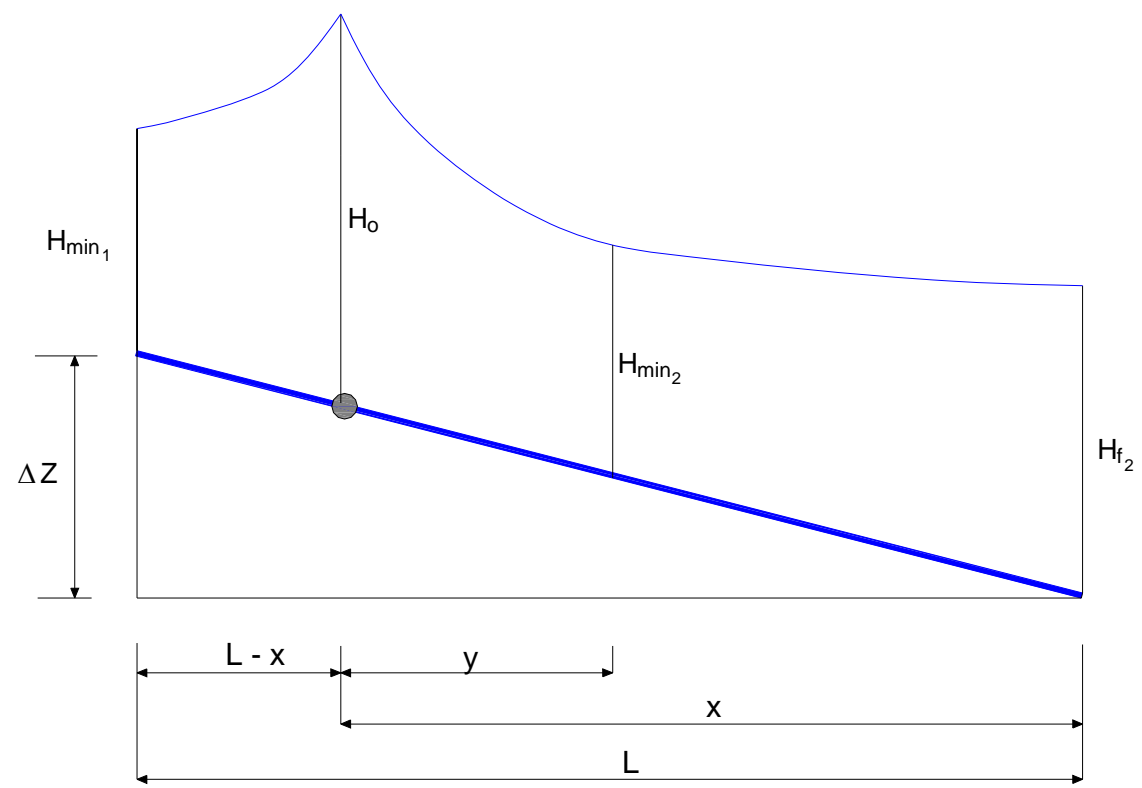

Figura 4: Localización del punto de alimentación intermedio en lateral o terciaria con desnivel.

La localización del punto de alimentación debe ser tal que la presión mínima en el tramo ascendente se iguale a la presión mínima en el tramo descendente.

Para la determinación del punto de alimentación se ha seguido el proceso que se indica en la siguiente figura. 


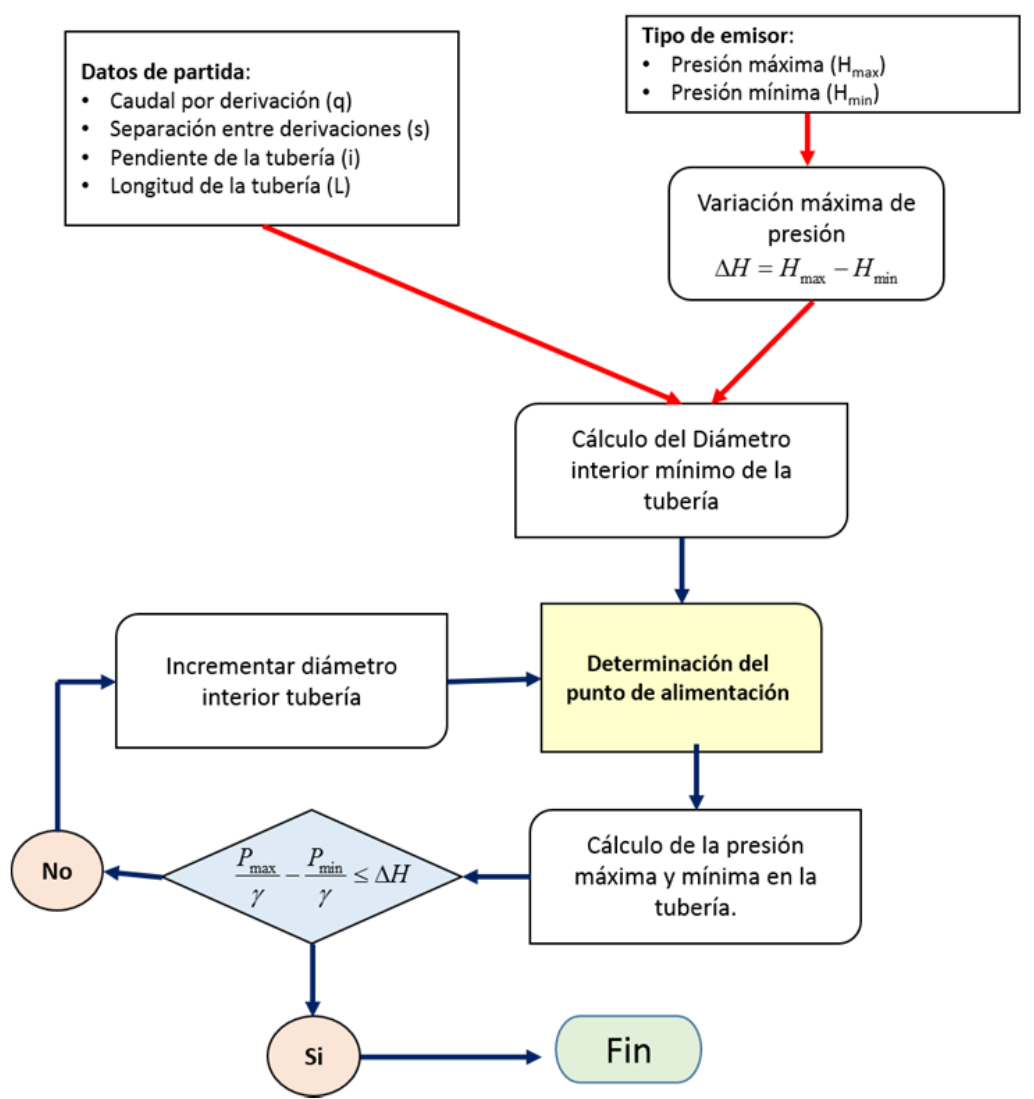

Figura 5:Esquema del proceso de determinación del punto de alimentación y dimensionado.

Se ha contemplado que las tuberías terciarias puedan ser telescópicas. En la figura se ilustra el diagrama de cálculo para este tipo de tuberías.

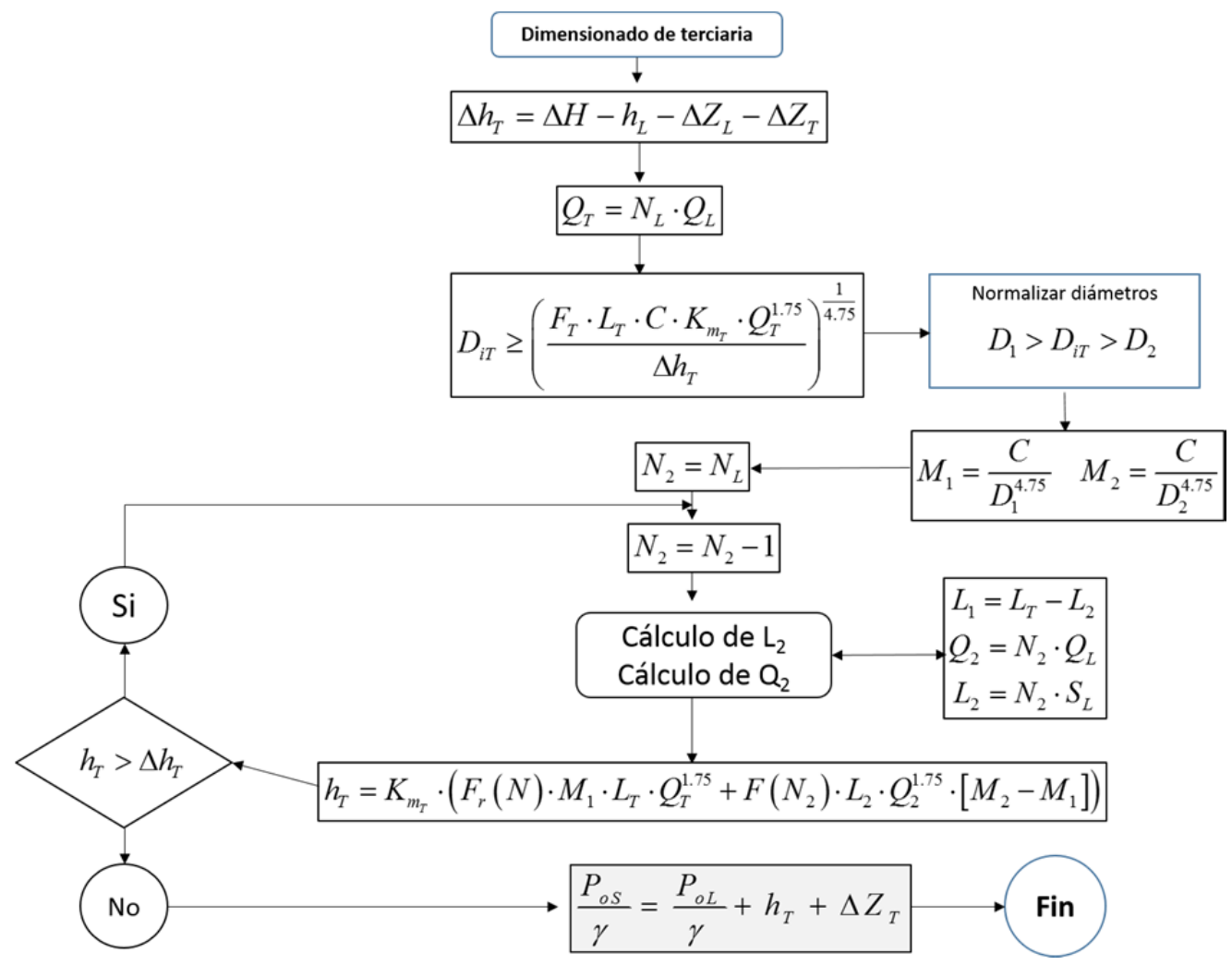

Figura 6: Proceso de cálculo de terciarias telescópicas. 


\section{Resultados}

El resultado final de la aplicación se presenta como un libro Excel habilitado para macros, aunque puede ser incorporado como complemento "Add-Ins" en el menú principal de Excel.

Al abrir el libro aparece una ventana de presentación.

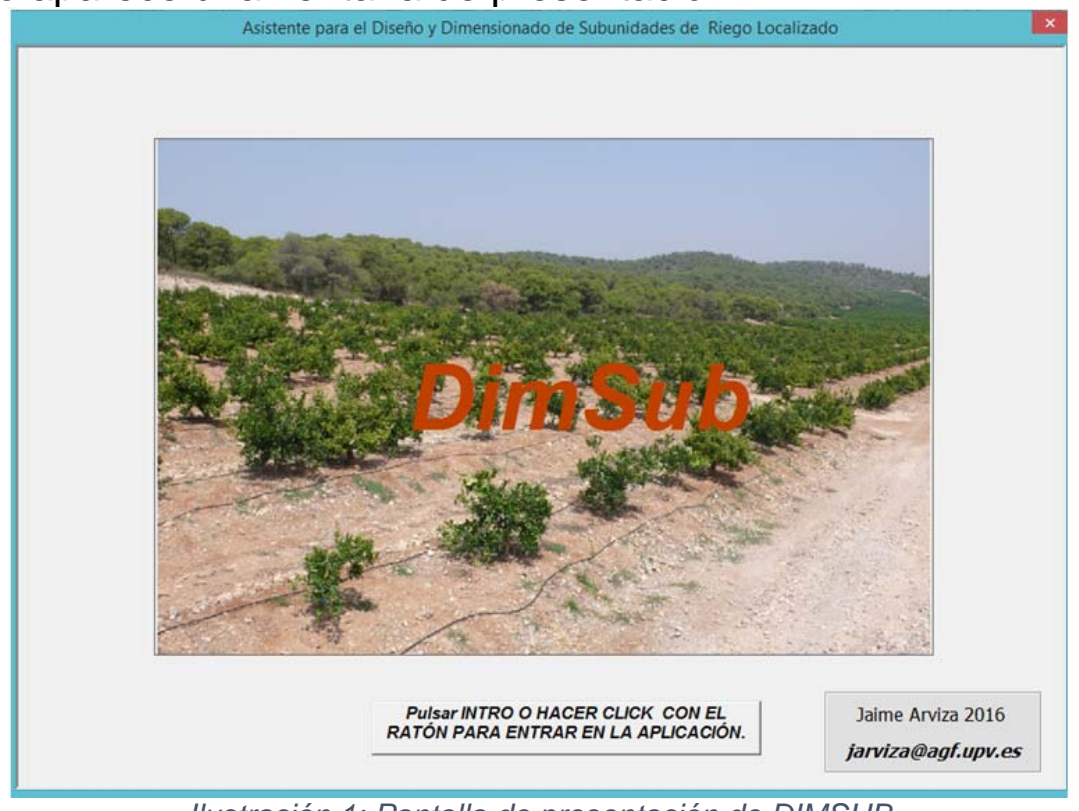

Ilustración 1: Pantalla de presentación de DIMSUB

Clicando en el botón de comando correspondiente se accede a la hoja activa desde la que se activan los formularios.

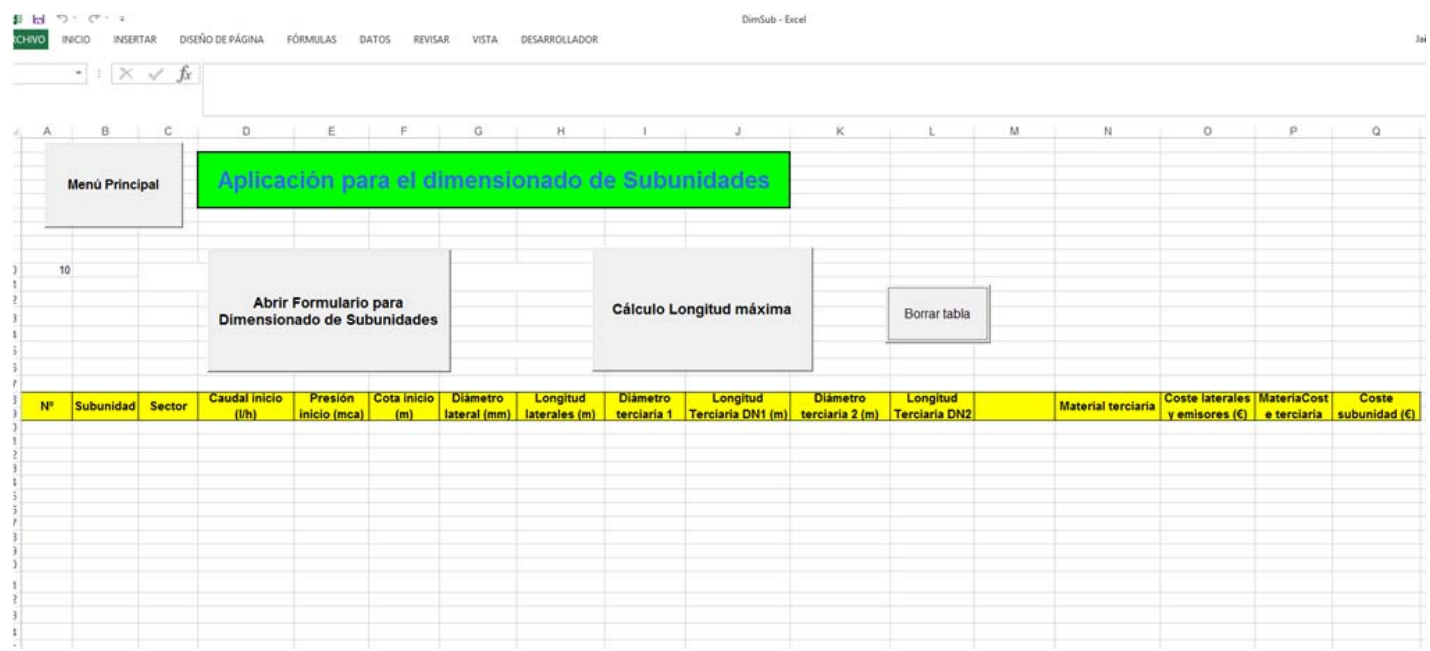

Ilustración 2: Hoja activa con los botones de comando con los formularios disponibles.

Si clicamos en "Abrir Formulario para dimensionado de Subunidades" aparece el mismo en pantalla. 


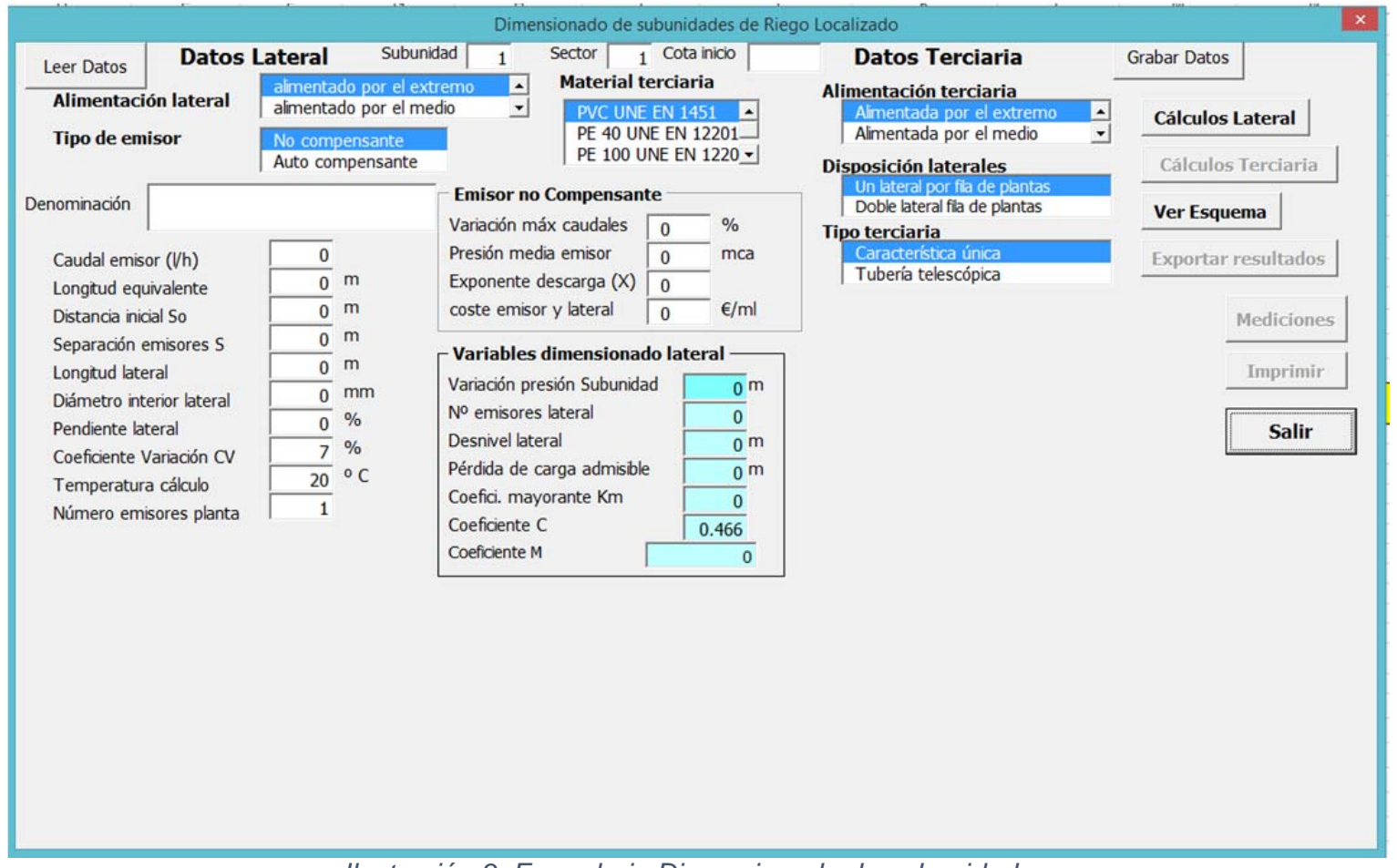

Ilustración 3: Formulario Dimensionado de subunidades.

Para ilustrar sus prestaciones y opciones de cálculo lo aplicaremos a un ejemplo concreto.

\section{Ejemplo}

Se trata del diseño y dimensionado de una subunidad de riego con emisores autocompesantes en una parcela con desniveles y cultivo de cítricos. El caudal del emisor es de $3.5 \mathrm{l} / \mathrm{h}$ con un rango de auto compensación de 10 a 40 mca. El emisor está integrado en una tubería lateral cuyo diámetro interior es de $146 \mathrm{~m}$. Los emisores están dispuestos a un metro en el lateral.

La superficie regable es de 11,67 has. El marco de plantación es de $6 \mathrm{~m} \times 4 \mathrm{~m}$ con doble lateral por fila de plantas. La separación entre laterales que alimentan a la misma fila es de $1 \mathrm{~m}$ y entre laterales que alimentan a filas adyacentes es de $5 \mathrm{~m}$. Las pendientes calculadas en las direcciones de lateral y terciarias son del $2 \%$ y 4 $\%$ respectivamente. 


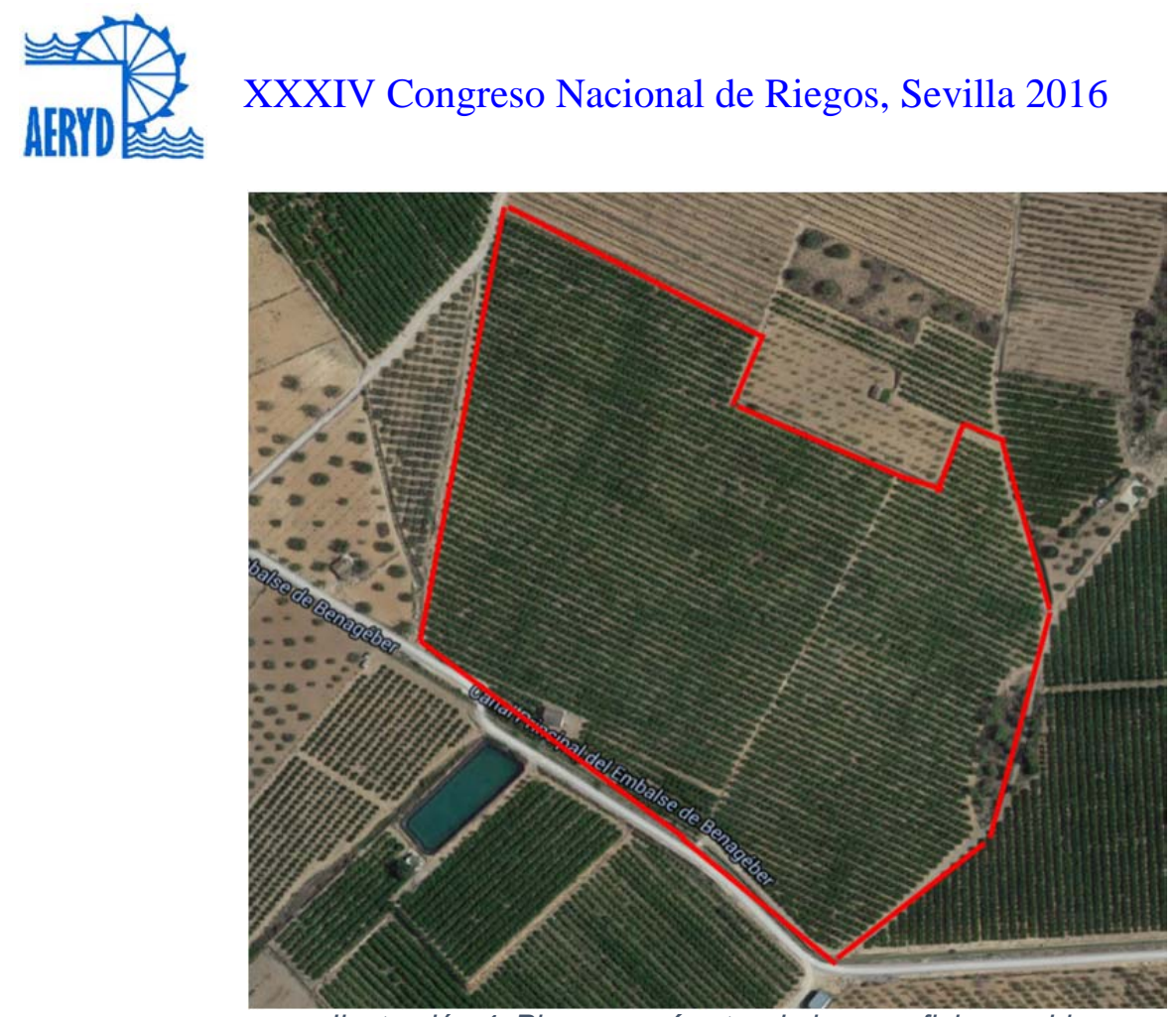

Ilustración 4: Plano y perímetro de la superficie regable
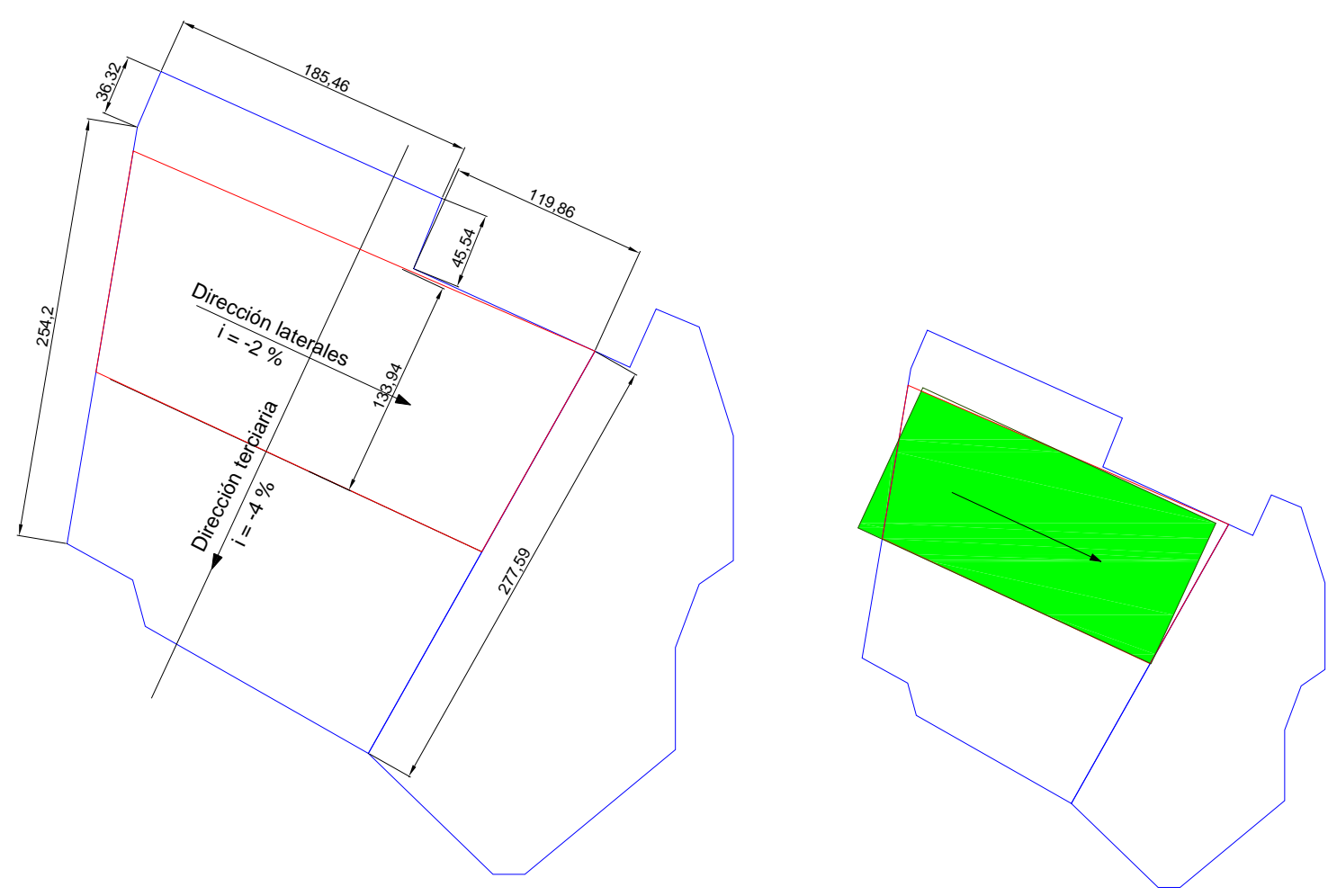

Ilustración 5: Cotas y dimensiones y subunidad a dimensionar

Una vez abierto el formulario se procede a la introducción de datos. Se acepta en primera instancia que los laterales se alimentarán por el extremo. Cuando procedo a calcular aparece la siguiente ventana de aviso. 


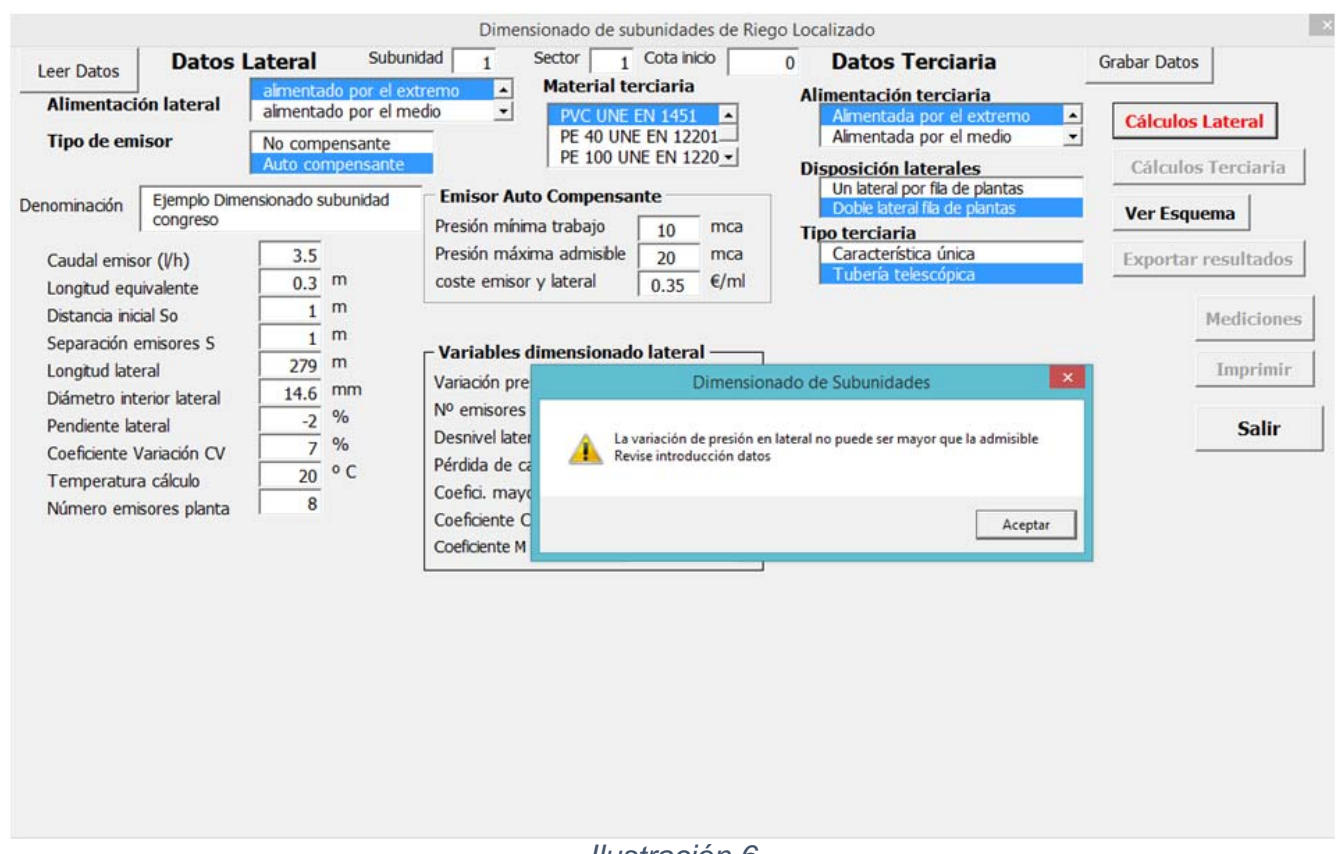

Ilustración 6

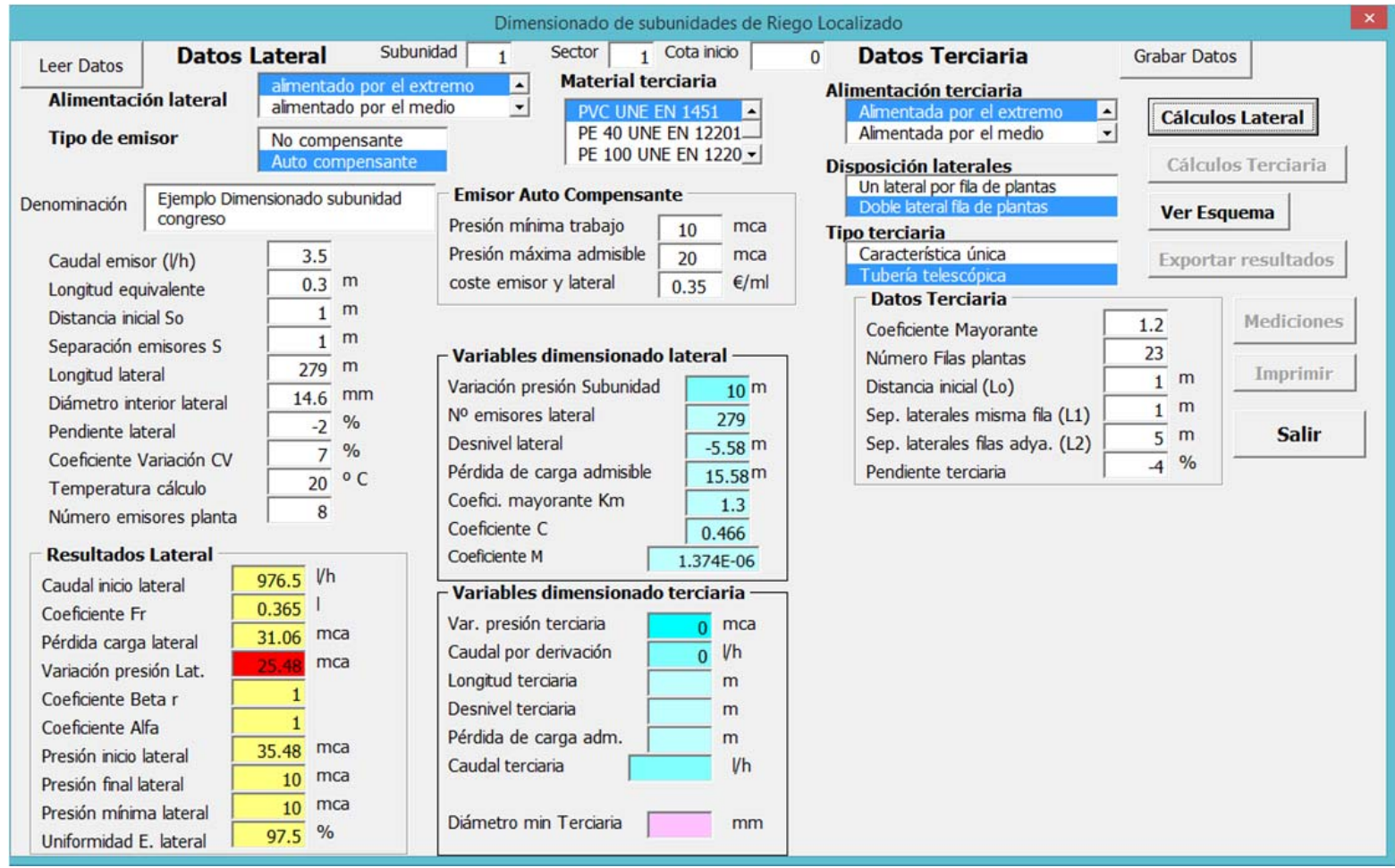

Ilustración 7: Dimensionado de lateral con exceso de variación de presión.

La aplicación me permite continuar los cálculos para ver el exceso de variación de presión en lateral y a qué es debido. La longitud es excesiva. Como la pendiente es significativa se opta por alimentar el lateral por un punto intermedio.

En este caso la variación de presión es admisible, tendré un tramo ascendente de longitud menor y otro descendente de mayor longitud cumpliendo que las presiones mínimas en ambos tramos son iguales, e iguales o mayores que la presión mínima admisible.

Podré proceder al dimensionado de la terciaria: 


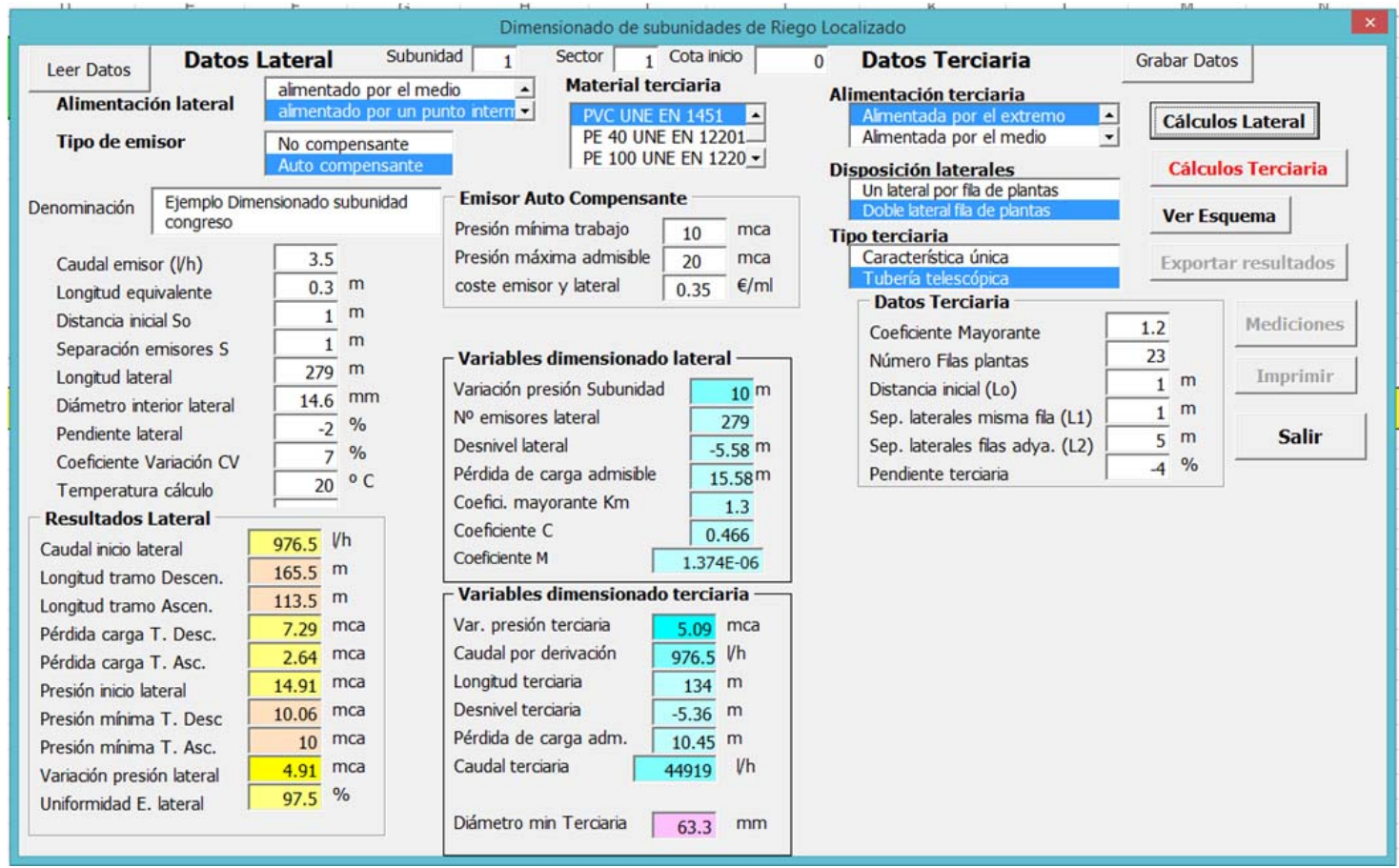

Ilustración 8: Resultados de la alimentación del lateral por el punto intermedio

En el dimensionado de la terciaria se ha optado por la solución de tubería telescópica.

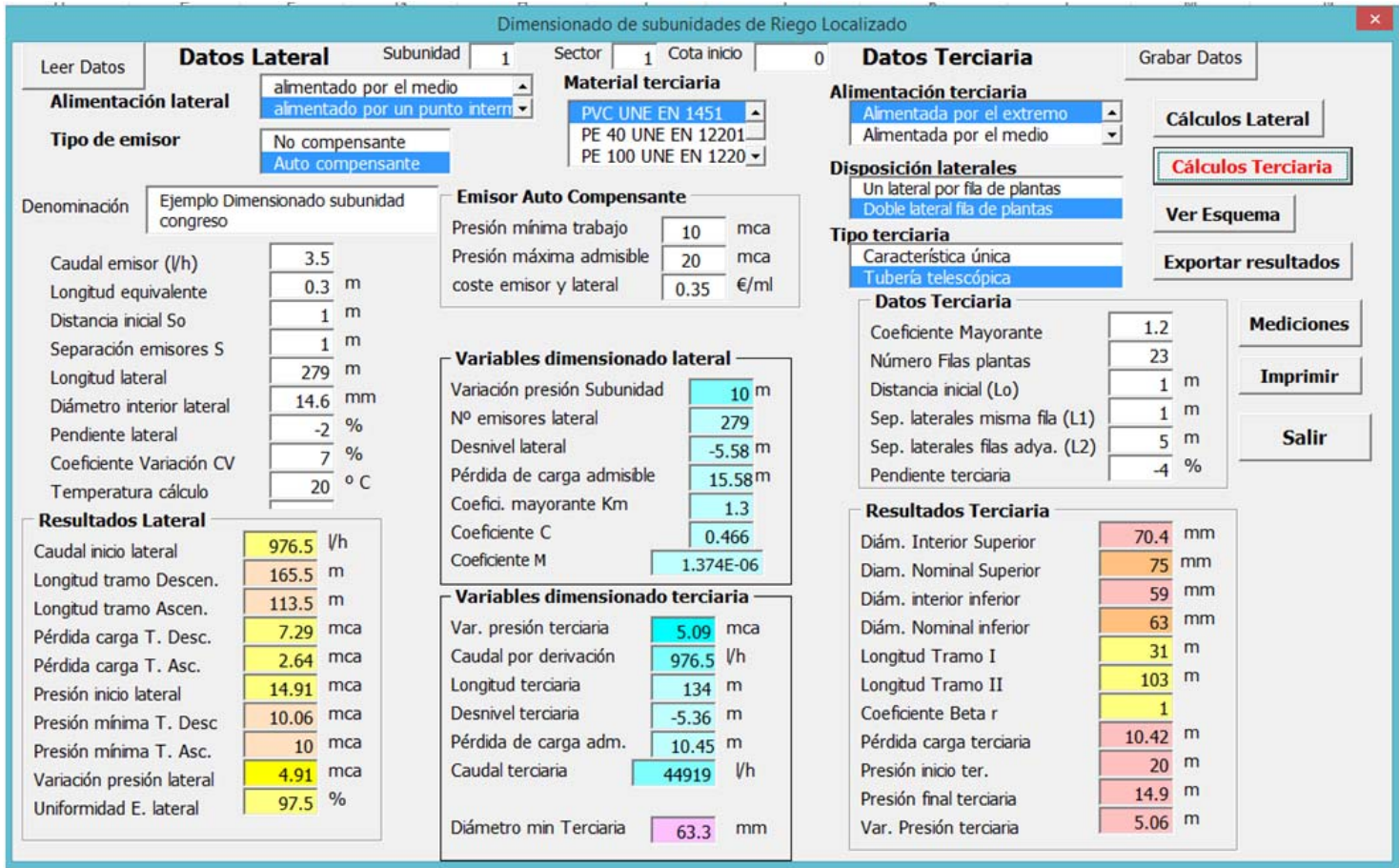

Ilustración 9: Resultados del dimensionado de la terciaria.

La aplicación permite la gestión de ficheros de datos desde el propio formulario, así como visualizar el esquema de cualquier tipo de alimentación de lateral y terciaria (Ilustración 10). Puede crearse una hoja de impresión para cada subunidad 
dimensionada y exportar resultados para su posterior tratamiento en el dimensionado de la red de transporte.

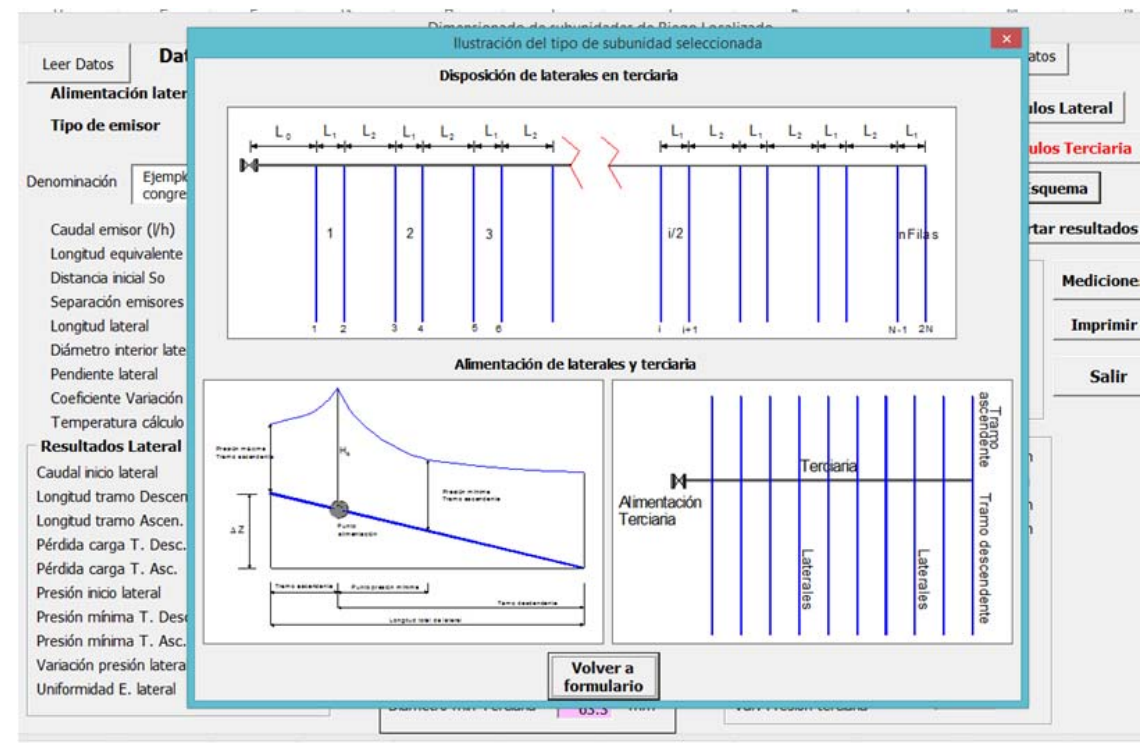

Ilustración 10

Finalmente, permite realizar para cada subunidad las mediciones de laterales, emisores y terciarias así como la correspondiente valoración económica.

\section{Conclusiones}

Dimsub es una herramienta de gran utilidad para el diseño de subunidades en proyectos de sistemas de riego localizado a presión.

Consideramos que la aplicación resulta de gran interés tanto en el ámbito técnico como académico, habiendo sido utilizada en el desarrollo de metodologías activas en la actividad docente con muy buenos resultados.

Como siguiente evolución de la aplicación, ésta debe ser integrada en RGW2015 y se está desarrollando un entorno gráfico propio para ampliar las prestaciones de la aplicación.

Al ser un software de distribución libre los autores están abiertos a cualquier tipo de sugerencias, mejoras y comentarios.

\section{Bibliografía}

Arviza, J. "Riego Localizado". Servicio Publicaciones Universitat Politècnica de València. 1996

Arviza, J et Al. "RG WIN 2015, Aplicación para el Diseño y Dimensionado de Redesde Riego a Presión en entorno Excel integrando parámetros agronómicos, energéticos e hidráulicos". XXXIII Congreso Nacional de Riegos. Universitat Politècnica de València. 2015 


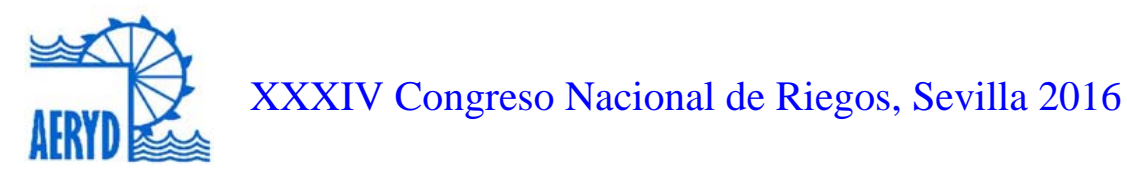

Kreyszig, Erwin. "Matemáticas avanzadas para ingenieros". Volumen 2. Ed. Limusa Willey. 2000

Montalvo López, T. "Riego Localizado. Diseño de instalaciones". Ediciones VJ. Valencia. 2005

Walkenbach,j "Excel 2010. Programación con VBA" Editorial Anaya. Madrid. 2010 